\title{
Article introductif
}

\section{Des évolutions marquantes pour le café en ce début de $x \mathrm{xI}^{\mathrm{e}}$ siècle}

\author{
André Charrier ${ }^{1}$ \\ Benoît Bertrand ${ }^{2}$ \\ Philippe Lashermes ${ }^{3}$ \\ ${ }^{1}$ Montpellier SupAgro \\ 2, place Pierre Viala \\ 34060 Montpellier cedex 2 \\ France \\ $<$ hacharrier@orange.fr> \\ ${ }^{2}$ Cirad \\ UMR RPB \\ (Cirad, IRD, université Montpellier II) \\ IRD \\ 911, avenue Agropolis \\ BP 64501 \\ 34394 Montpellier cedex 5 \\ France \\ $<$ benoit.bertrand@cirad.fr $>$ \\ ${ }^{3}$ IRD \\ UMR RPB \\ (Cirad, IRD, université Montpellier II) \\ IRD \\ 911, avenue Agropolis \\ BP 64501 \\ 34394 Montpellier cedex 5 \\ France \\ <philippe.lashermes@ird.fr>
}

A u cours du $x^{e}$ siècle, la production et les marchés des cultures tropicales de rente, dont le café, ont subi d'importantes évolutions, en rapport avec les indépendances des pays africains et asiatiques et la mondialisation des marchés des produits agricoles. Concrètement, la géographie mondiale de la production et de la consommation du café s'est transformée. Le Brésil ( 40 \% de la production mondiale) et l'Amérique latine, producteurs historiques du café arabica, continuent de dominer le marché. À l'opposé, le Vietnam est devenu cette dernière décennie le premier producteur de café robusta et le deuxième producteur mondial, avec des pratiques agronomiques intensives, probablement peu durables. Dans le même temps, la production d'autres pays africains a décliné avec la chute des cours et l'instabilité politique (Angola, Côte d'Ivoire, Madagascar...).

La production mondiale de café vert provenant de plus de 60 pays atteint aujourd'hui 8 millions de tonnes (2/ 3 arabica, 1/3 robusta), à comparer à un million de tonnes presque exclusivement d'arabica en 1900, produit aux Amériques par l'espèce Coffea arabica. Le développement de la caféiculture dans les empires coloniaux d'Afrique et d'Asie a été largement fondé sur l'espèce Coffea canephora produisant le robusta, mieux adaptée aux régions tropicales chaudes et humides. Les revenus d'une centaine de millions de personnes et la balance commerciale de nombreux pays du Sud dépendent encore des exportations de café vers les pays consommateurs du Nord. Le café qui occupe le troisième rang du marché mondial, a toujours été affecté par de fortes fluctuations de la production et des prix malgré les tentatives de régulation comme les marchés à terme puis l'accord international de 1960. Le courant de libéralisation et la mondialisation des marchés des années 1990 ont entraîné pour le café une chute marquée des prix et des investissements nécessaires à la modernisation des plantations. La reprise actuelle s'accompagne d'une diversification des productions et des marchés (cafés gourmets, café équitable, café biologique, marques commerciales...) (Daviron et Ponte, 2007).

La consommation dans le monde s'est accrue régulièrement de $2 \%$ par an dans les trente dernières années, avec une diversification des présentations (cafés en grains ou soluble, café décaféiné, café "expresso " en capsules...). L'attrait du café a gagné de nouveaux pays consommateurs comme le Japon ou la Russie, mais aussi, pour leur consommation domestique, les populations des pays producteurs comme le Brésil, l'Indonésie...

L'état des connaissances et des recherches scientifiques dans les différents domaines de la filière café fait régulièrement l'objet d'ouvrages de synthèse (Illy et Viani, 2005; Clarke et Vitzthum, 2008 ; Wintgens, 2009) et de communications dans des forums nationaux et internationaux, tout particulièrement lors des colloques bisannuels de l'Association pour la science et l'information sur le café (ASIC) organisés avec les fédérations de producteurs et les industriels. Ainsi, ce numéro thématique des Cabiers Agricultures dévolu au café a pour objectif d'illustrer quelques-unes des évolutions remarquables concernant cette plante stimulante et sa filière en ce début de $\mathrm{xxI}^{\mathrm{e}}$ siècle, au regard des 
enjeux pour l'agriculture, l'environnement et la santé.

\section{Impact}

des changements climatiques

\section{sur la caféiculture et la production durable}

Différentes études sur l'impact des changements climatiques ont surtout permis de modéliser l'évolution de la distribution spatiale et altitudinale des zones de culture favorables à la principale espèce cultivée $C$. arabica à l'échelle régionale et de simuler leur déplacement prévisible (Läderach et al., 2008). Des recherches prospectives pour une production durable sont d'autant plus nécessaires que le renouvellement des plantations d'arbres fruitiers pérennes tels que les caféiers est réalisé tous les 20 à 30 ans, voire plus.

En outre, l'intensification de la caféiculture (monoculture à forte densité, sans ombrage, avec intrants chimiques et irrigation, mécanisation de la récolte) s'est développée ces 50 dernières années avec des variétés à haut potentiel, mais elle est remise en cause pour son impact environnemental. D'autres systèmes de culture innovants peuvent limiter les effets du changement climatique sur la production et les maladies, en incluant aussi des variétés hybrides F1 plus adaptées (Bertrand et al., 2012). Leur diffusion introduit une nouvelle diversité génétique compatible avec des plantations productives et durables, en système agroforestier favorable à la protection de l'environnement. Le retour à des pratiques d'agroforesterie (culture sous ombrage avec converture du sol et variétés rustiques) réduit l'impact des stress et les problèmes sanitaires (Mouen Bedimo et al., 2012), avec un équilibre écologique plus favorable (présence de pollinisateurs et de prédateurs naturels, enrichissement en matière organique et microfaune $d u$ sol...), et la prise en compte des services écosystémiques (Rapidel et al., 2011). Les travaux réalisés sur cette approche en Amérique centrale, en Inde et Afrique orientale sont consultables sur le site de Cafnet ${ }^{1}$.

\section{Conservation}

\section{de la biodiversité caféière}

Les changements globaux ont aussi un impact sur la biodiversité et la disparition des espèces. Pour les caféiers regroupés dans le genre Coffea (Davis et al., 2006) et originaires des forêts tropicales d'Afrique et d'Asie, des équipes internationales et françaises (Cirad, IRD et Museum national d'histoire naturelle) s'étaient déjà investies depuis les années 1960 dans l'inventaire et la constitution de conservatoires de ces caféiers spontanés et cultivés menacés par la déforestation. Deux problèmes majeurs restaient à résoudre pour atteindre l'objectif d'une conservation durable :

- la conservation ex situ dans des conservatoires de plantes vivantes ou de banques de semences soustrait les caféiers aux processus évolutifs et adaptatifs naturels; pour ce faire, il est indispensable de favoriser les projets de gestion in situ des forêts naturelles abritant des caféiers spontanés comme à Madagascar et en Éthiopie, et aussi de maintenir en culture des variétés locales de C. arabica comme en Éthiopie (Labouisse et Adolphe, 2012) ;

- contrairement aux graines des espèces végétales dont les semences déshydratées sont habituellement conservées sur le long terme au froid, celles des caféiers perdent rapidement leur viabilité ; des études récentes sur leur biologie ouvrent la voie à la conservation de longue durée des ressources génétiques caféières par cryoconservation, ainsi que la conservation de courte durée des semences des variétés à diffuser (Dussert et al., 2012).

\section{Avancées en biologie et innovations}

Les progrès scientifiques réalisés au cours de la dernière décennie en génétique-génomique, physiologie et

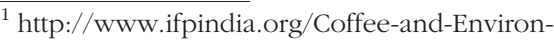
mental-Services-in-the-Western-Ghats.html
}

biotechnologies chez les caféiers trouvent des domaines d'application et d'innovation illustrés par quelques exemples :

- la maîtrise de l'embryogenèse somatique en milieu liquide permet la multiplication massive de variétés clonales sélectionnées (hybrides F1 ou individus élites) à structures génétiques hétérozygotes, non reproductibles par graines ; ce procédé permet la diffusion industrielle d'hybrides F1 de C. arabica en Amérique centrale (Etienne et al., 2012) ;

- les nombreux composés biochimiques du café vert qui jouent un rôle majeur dans la qualité du café-boisson sont connus ; les déterminants génétiques et moléculaires des voies de biosynthèse des principaux composés sont aujourd'hui accessibles par la génomique fonctionnelle, ainsi que les facteurs modifiant leur expression chez le caféier Arabica (Joët et al., 2012);

- l'analyse de la diversité des espèces de caféiers au niveau moléculaire a fait progresser la connaissance de leur structuration en groupes génétiques, de leur phylogénie et de l'histoire évolutive du genre Coffea. En outre, le séquençage du génome complet de l'espèce $C$. canephora sera finalisé en 2012 par un consortium international de recherche et ouvrira la voie à des applications diversifiées. L'amélioration génétique pour la résistance aux maladies des caféiers et pour la qualité du café en sera grandement renouvelée (Lashermes et al., 2012).

- les choix stratégiques qui permettent d'utiliser au mieux ces connaissances biologiques et les ressources génétiques et génomiques au service de l'innovation variétale sont présentés pour l'espèce C. canephora (Montagnon et al., 2012).

\section{Nouvelles tendances du marché mondial du café et de I'organisation des producteurs}

Les dernières évolutions marquantes du marché concernent l'initiative du 
commerce équitable et la différenciation des produits certifiés, avec le développement de marchés de niches et la tendance à des standards de durabilité, en référence aux trois piliers du développement durable. La part de ce marché est passée de $1 \%$ en 2000 à $8,60 \%$ en 2009. Les nombreuses labellisations proposées sont conçues pour une diversité d'objectifs (environnement, socioéconomique, qualité) et de groupes cibles (producteurs, consommateurs, industrie). Les implications de cette transition vers la diversification des produits primaires tels que le café sont analysées du triple point de vue de la répartition de la plus-value, de la transparence et de la substitution des lots dans la filière (Vagneron et Daviron, 2012).

En particulier, les paysans et leurs organisations (OP) jouent un rôle primordial dans le développement et la gestion des certifications. Les choix de certification sont de nature à redessiner le paysage de la caféiculture (Faure et al., 2012).

Pour accéder aux marchés à forte valeur ajoutée des cafés spéciaux, il est indispensable de caractériser les terroirs favorables à l'obtention d'un produit de typicité reconnue. Ainsi une typologie sensorielle des cafés produits en République dominicaine a été établie et permet la localisation des terroirs aux conditions les plus favorables (Aguilar et al., 2012). Cette approche pourrait être étendue à bien d'autres territoires.

Enfin les stratégies adoptées par les petits producteurs permettent le maintien de productions paysannes assurant la multifonctionnalité et le rôle patrimonial des plantations de caféiers en Afrique (Sibelet et Montzieux, 2012).

\section{Risques associés}

\section{à la consommation du café}

Un autre aspect de la qualité est, du point de vue des consommateurs, la qualité sanitaire du café et la relation café-santé.

D'une part, des sources multiples de contaminants aux différentes étapes de la filière peuvent avoir un impact sur la santé : intrants chimiques uti- lisés en caféiculture, résidus de pesticides, mycotoxines (OTA) produites par des moisissures et hydrocarbures aromatiques polycycliques (HAP). Ces contaminants font l'objet d'une attention croissante et de réglementations aux États-Unis et en Europe, même si la production de cafés provenant de l'agriculture biologique se développe (Durand et al., 2012).

D'autre part, le café est une boisson consommée pour son action stimulante associée à la caféine, mais il est régulièrement mis en cause dans ses effets physiologiques et dans de nombreuses pathologies humaines. L'exploitation d'une grande diversité d'études par des méta-analyses permet aujourd'hui d'avoir une vue globale des conditions de consommation de cette boisson pour un effet bénéfique sur le plan santé (Nehlig, 2012).

\section{Conclusion}

En guise de conclusion, nous tenterons une prospective dans plusieurs domaines de la filière café.

- Les prévisions de consommation sont à la hausse au niveau mondial, en particulier avec l'augmentation de la consommation des pays émergents et de la consommation domestique des pays producteurs. De ce fait, cet accroissement devrait surtout concerner la demande en robusta/soluble. La production mondiale s'adaptera, d'autant que les zones de culture favorables à $C$. arabica vont se déplacer et se restreindre, conséquence du changement climatique plus favorable à $C$. canephora. En conséquence, la répartition géographique des régions de production du café va changer à l'échelle du xxI ${ }^{\mathrm{e}}$ siècle, sous le double impact du marché et du climat. La réduction des zones favorables à l'Arabicaculture devrait entraîner une augmentation des prix des cafés de qualité produits dans les zones de haute altitude.

- Les rapports entre les acteurs de la filière café ont récemment évolué avec le développement du commerce équitable qui a promu des relations plus étroites entre les consommateurs et des producteurs clairement identifiés. Cette diversification des cafés par leur origine et leurs conditions de production associe largement les groupements de producteurs et les industriels du café à des démarches de labellisation variées. L'avenir et l'extension de ces marchés de niche dépendront de la juste répartition de la plus-value entre les acteurs.

- Pour une culture de l'importance économique du café, la faiblesse des recherches est flagrante, en particulier en amélioration variétale. Dans le secteur public, elle résulte du manque de ressources humaines et de capacités de financement des pays du Sud (à l'exception du Brésil et de la Colombie). Quant à la production de semences améliorées par le secteur privé, elle ne s'est pas développée, en l'absence de protection juridique des variétés créées et de rentabilité des investissements. Le contexte est en cours de changement avec l'adoption par les pays du Sud de législations ad hoc susceptibles de favoriser la synergie public/privé en recherche. Il en est de même, de la multiplication clonale par biotechnologie : elle requiert des investissements hors de portée des paysans, mais elle peut s'avérer rentable pour le secteur privé et contribuer à la création d'un secteur semencier professionnel qui proposera un choix plus grand de variétés. - Enfin, la conservation et l'accès aux ressources génétiques caféières restent des défis majeurs pour les générations futures. La survie des espèces sauvages de caféiers est directement dépendante de la mise en place de plans nationaux et internationaux de conservation de leurs habitats naturels, les forêts tropicales d'Afrique. Pour les deux espèces cultivées, la diversité est surtout assurée dans les plantations des caféiculteurs des zones d'origine et dans les collections des centres de recherche. Au cours des deux derniers siècles, ces ressources ont fait l'objet de transferts et d'échanges à l'échelle planétaire. Aujourd'hui, leur acquisition va être dépendante de la mise en place progressive de législations nationales plus ou moins restrictives réglementant l'accès aux ressources génétiques et le partage des avantages.

\section{Références}

Aguilar P, Ribeyre F, Escarramán A, Bastide P, Berthiot L, 2012. Les profils sensoriels des cafés sont liés aux terroirs en République dominicaine. Cahiers Agricultures 21 : 169-78. doi : 10.1684/ agr.2012.0546 
Bertrand B, Montagnon C, Georget F, Charmetant $P$, Etienne $H, 2012$. Création et diffusion de variétés de caféiers Arabica : quelles innovations variéta-

les ? Cahiers Agricultures 21: 77-88. doi : 10.1684/agr.2012.0547

Clarke R, Vitzthum O, 2008. Coffee: Recent Developments. New York : John Wiley \& Sons.

Daviron B, Ponte S, 2007. Le paradoxe du café. Paris : éditions Quae.

Davis AP, Govaerts R, Bridson SM, Stoffelen P, 2006. An annotated taxonomic conspectus of the genus Coffea (Rubiaceae). Botanical Journal of the Linnean Society 152 : 465-512.

Durand N, Gueule D, Fourny G， 2012. Les contaminants du café. Cahiers Agricultures 21 : 192-6. doi : 10.1684/agr.2012.0551

Dussert S, Couturon E, Engelmann F, Joët T, 2012 Biologie de la conservation des semences de caféiers : aspects fondamentaux et conséquences pratiques. Une revue. Cahiers Agricultures 21 106-14. doi : 10.1684/agr.2012.0552

Etienne $\mathrm{H}$, Bertrand $\mathrm{B}$, Montagnon $\mathrm{C}$, Bobadilla Landey R, Dechamp E, Jourdan I, et al., 2012. Un exemple de transfert de technologie réussi dans le domaine de la micropropagation : la multiplication de Coffea arabica par embryogenèse somatique. Cahiers Agricultures 21 : 115-24. doi : 10.1684/ agr.2012.0553

Faure G, Le Coq JF, Vagneron I, Hocdé H, Muñoz GS, Kessari M, 2012. Stratégies des organisations de producteurs de café au Costa Rica vis-à-vis des certifications environnementales et sociales. Cahiers Agricultures 21 : 162-8. doi : 10.1684/ agr.2012.0545

Illy A, Viani R., 2005. Espresso coffee : the science of quality. 2nd edition. London : Elsevier Academic Press.

Joët T, Pot D, Ferreira LP, Dussert S, Marraccini P, 2012. Identification des déterminants moléculaires de la qualité du café par des approches de génomique fonctionnelle. Une revue. Cahiers Agricultures 21 : 125-33. doi : 10.1684/ agr. 2012.0548

Labouisse JP, Adolphe C, 2012. Conservation et gestion des ressources génétiques du caféier Arabica (Coffea arabica L.) : un défi pour l'Éthiopie. Cahiers Agricultures 21 : 98-105. doi : 10.1684/ agr.2012.0554

Laderach P, Jarvis A, Ramírez J, Fisher MJ, 2008. Predictions of land use changes under progressive climate change in coffee growing regions of the AdapCC project: Final report. Chiapas, Mexico, Cali, Colombia: October 2008 [on line]. Cali (Colombia): Centro Internacional de Agricultura Tropical (CIAT).

Lashermes $\mathrm{P}$, Combes MC, Dereeper A, Cenci A, 2012. Diversité et évolution des caféiers à la lumière de la génomique. Cahiers Agricultures 21 : 134-42. doi : 10.1684/agr.2012.0555
Montagnon C, Cubry P, Leroy T, 2012. Amélioration génétique du caféier Coffea canephora Pierre : connaissances acquises, stratégies et perspectives. Cahiers Agricultures 21: 143-53. doi : 10.1684/agr.2012.0556

Mouen Bedimo JA, Dufour B, Cilas C, Avelino J, 2012. Effets des arbres d'ombrage sur les bioagresseurs de Coffea arabica. Cahiers Agricultures 21 : 89-97. doi : 10.1684/agr.2012.0550

Nehlig A, 2012. Effets physiologiques du café et santé humaine. Une revue. Cahiers Agricultures 21 : 197-207. doi : 10.1684/agr.2012.0549

Rapidel B, Le Coq JF, Beer J, eds., 2011. Ecosystem services from agriculture and agrofores try : measurement and payment. Londres : Earthscan Publications.

Sibelet N, Montzieux M, 2012. Les facteurs de résilience de la caféiculture au Kenya : de la sécurisation alimentaire à la retraite. Cahiers Agricultures 21: 179-91. doi : 10.1684/ agr.2012.0563

Vagneron I, Daviron B, 2012. Le café dans la jungle des standards de durabilité environnementale et sociale. Cahiers Agricultures 21: 154-61. doi : 10.1684/agr.2012.0557

Wintgens JN, ed., 2009. Coffee: growing, processing, sustainable production. Weinheim (Germany): Wiley-VCH Verlag. 